\title{
Determinação de constantes de transferência de compostos voláteis em lagoas de tratamento de esgoto
}

\section{Determination of transfer constants of volatile compounds in sewage treatement ponds}

Data de entrada: 02/06/2019

Data de aprovação: $18 / 06 / 2020$

Carlos Eduardo Pereira de Morais ${ }^{1}$ | Silvânia Lucas dos Santos² | Adrianus van Haandel ${ }^{1 *}$

DOI: https://doi.org/10.36659/dae.2021.018

ORCID ID

Morais CEP (D) https://orcid.org/0000-0002-6910-9579

Santos SL iD https://orcid.org/0000-0002-6905-3966

Haandel A (D) https://orcid.org/0000-0002-9937-6715

\section{Resumo}

Em lagoas que tratam águas residuárias, há componentes voláteis em intercâmbio com gases na atmosfera. Os principais componentes são dióxido de carbono, oxigênio dissolvido e amônia. O intercâmbio desses compostos com o ar é importante porque afeta o pH e a disponibilidade de oxigênio dissolvido em lagoas de polimento. Uma investigação experimental demonstrou que a cinética de transferência dos compostos é regida pela lei de Fick; esta afirma que a taxa de transferência é proporcional ao déficit, ou seja, há diferença entre a concentração do composto volátil dissolvido e sua concentração de saturação. A constante de transferência foi determinada experimentalmente e a influência da temperatura e da profundidade foi estabelecida. Em soluções supersaturadas, os componentes tendem a se desprender como bolhas de gás, mas esse desprendimento não se verifica para concentrações normalmente encontradas para $\mathrm{O}_{2}, \mathrm{CO}_{2}$ e $\mathrm{NH}_{3}$ em lagoas de tratamento de esgoto.

Palavras-chave: Lagoas de polimento. Cinética de transferência. Compostos voláteis.

\section{Abstract}

In wastewater treatment ponds, volatile components are exchanged with gases in the atmosphere. The main components are carbon dioxide, dissolved oxygen and ammonia. The exchange of these compounds with air is important, because they affect the $\mathrm{pH}$ and the availability of dissolved oxygen in polishing ponds. An experimental investigation showed that the transfer kinetics of compounds is governed by Fick's law, that states that the transfer rate is proportional to the deficit, which is the difference between the concentration of the dissolved volatile compound and the saturation concentration. The transfer constant was determined experimentally, and the influence of temperature and depth was established. In supersaturated solutions the components tend to desorb like gas bubbles, but this desorption does not occur at concentrations normally found for $\mathrm{O}_{2}, \mathrm{CO}_{2}$ and $\mathrm{NH}_{3}$ in ponds for sewage treatment.

Keywords: Polishing ponds. Volatile compounds. Transfer kinetics.

\footnotetext{
${ }^{1}$ Universidade Federal de Campina Grande - UFCG - Campina Grande - Paraíba - Brasil.

2 Universidade Federal do Rio Grande do Norte - UFRN - Natal - Rio Grande do Norte - Brasil.

"Autor correspondente: adrianusvhagmail.com.
} 


\section{INTRODUÇÃO}

Em lagoas de tratamento de águas residuárias há intercambio com a atmosfera de compostos voláteis: dióxido de carbono $\left(\mathrm{CO}_{2}\right)$, amônia $\left(\mathrm{NH}_{3}\right)$, oxigênio dissolvido (OD), gás sulfídrico $\left(\mathrm{H}_{2} \mathrm{~S}\right)$ e metano $\left(\mathrm{CH}_{4}\right)$. Esses compostos são gases e têm uma solubilidade limitada em água, por isso tende-se a estabelecer na fase líquida uma concentração que está em equilíbrio com a concentração do gás no ar: a concentração de saturação. Se a concentração do gás dissolvido é temporariamente menor que a de saturação, haverá absorção do gás pela fase líquida; caso contrário haverá transferência para a atmosfera. No caso de amônia, gás sulfídrico e metano, não há uma presença mensurável no ar, de modo que ocorre apenas a dessorção desses gases. No caso de $\mathrm{CO}_{2}$ e $\mathrm{OD}$, pode haver absorção ou dessorção, dependendo de se a sua concentração na lagoa está sub ou supersaturada em relação à concentração de saturação.

O intercâmbio de alguns componentes voláteis entre a lagoa e a atmosfera é importante, porque afeta diretamente vários parâmetros na lagoa. Outros, como metano e gás sulfídrico não afetam mensuravelmente os parâmetros, mas têm sua importância: o metano é um gás que contribui para o efeito estufa, 21 vezes mais danoso que o $\mathrm{CO}_{2}$ (YVON-DUROCHER et al., 2014), e o gás sulfídrico é o responsável principal por odores ofensivos que lagoas podem exalar. Contudo, como esses componentes não têm efeito sobre as variáveis nas lagoas, não serão considerados neste trabalho.

No caso do OD, a absorção de oxigênio que ocorre enquanto a lagoa está subsaturada ajuda a manter o ambiente aeróbio, o que facilita a produção de um efluente com baixo teor de material orgânico biodegradável. Se uma lagoa recebe um efluente com uma concentração elevada de material orgânico, a taxa de oxidação (consumidora de OD) será grande em comparação com a taxa de fotossíntese (produtora de OD). Nessas condições, a concentração de OD tende a ser baixa, mas poderá ser mantida uma concentração de $O D$ pelo processo de absorção, apesar do consumo líquido de OD na lagoa. Caso a absorção de OD não seja suficiente para manter oxigênio dissolvido na lagoa, um ambiente anaeróbio se estabelecerá e a remoção do material orgânico procederá via digestão anaeróbia. Se na lagoa a taxa de fotossíntese superar a de oxidação, a produção biológica de oxigênio e a concentração de OD tendem a aumentar, possivelmente chegando acima da concentração de saturação, o que levará à dessorção de OD. Na supersaturação, poderá haver formação de bolhas de oxigênio que emergem da fase líquida e na sua trajetória ascendente tendem a se agregar a flocos de algas, formando assim uma camada superficial de sólidos sobre a lagoa, prejudicando a transparência e, portanto, o processo de fotossíntese na lagoa.

No caso de $\mathrm{CO}_{2}$ e $\mathrm{NH}_{3}$, o intercâmbio causa variação do $\mathrm{pH}$. Em ambiente anaeróbio, $\mathrm{C}_{2}$ normalmente encontra-se supersaturado (equilíbrio com o biogás e a consequente dessorção tende a aumentar o $\mathrm{pH}$ (VAN HAANDEL; LETTIN$\mathrm{GA}, 1994)$. A transferência de $\mathrm{NH}_{3}$ é equivalente à adição de um ácido forte: aumenta a acidez e diminui a alcalinidade de modo que o $\mathrm{pH}$ tende a diminuir (PIVELI; KATO, 2006).

Normalmente é de interesse aumentar o pH em lagoas para que se torne viável a remoção de nutrientes (SANTOS; VAN HAANDEL, 2021). A remoção de nutrientes em lagoas de tratamento é particularmente importante se o efluente está sendo tratado para ser lançado em águas de superfície, o que geralmente acontece, principalmente nas regiões do interior do país. A remoção de nutrientes de lagoas de tratamento pelo aumento do $\mathrm{pH}$ na prática é a alternativa mais plausível para evitar o processo de eutrofização que está se observando em quase todas as águas de superfície no país. 
A supersaturação de $\mathrm{CO}_{2}$ e $\mathrm{NH}_{3}$ nas lagoas de polimento também pode formar bolhas de gás que se desprendem da fase líquida e a concentração para que tal fenômeno ocorra é investigada no presente trabalho.

\subsection{Modelo de intercâmbio de componentes voláteis}

A taxa de transferência de um componente volátil dissolvido num líquido em contato com uma fase gasosa pode ser descrita pela lei de Fick, que expressa que a taxa é proporcional ao grau de supersaturação na fase líquida:

$r_{d}=(d C / d t)_{a}=k_{d}\left(C_{s}-C\right)$

Em que:

$r_{d}=(d C / d t)_{a}=$ taxa de transferência de C para a fase gasosa $(\mathrm{mg} / \mathrm{L} / \mathrm{h})$;

$k_{d}=$ constante de transferência $\left(h^{-1}\right)$;

$\mathrm{C}_{\mathrm{s}}=$ concentração de saturação do componente volátil (mg/L);

$\mathrm{C}=$ concentração momentânea do componente volátil na superfície do líquido (mg/L).

A Eq. 1 mostra que não há transferência $\left(r_{d}=0\right)$ quando a concentração do componente volátil é igual à concentração de saturação, que é a concentração de equilíbrio entre as fases líquida e gasosa (o ar). Se a concentração do componente (C) na fase líquida for menor que a concentração de saturação (Cs), a taxa de transferência é negativa: a solução é subsaturada e há absorção de modo que a concentração do componente dissolvido aumenta. Se a concentração for supersaturada, a taxa de transferência é negativa: há transferência do componente volátil da fase líquida para a atmosfera, que é expressa pela mesma Eq. 1. Todavia, se a concentração dos compostos voláteis for muito maior que a saturação, a lei de Fick deixa de ser válida, e os compostos se desprendem como bolhas de gás subindo na fase líquida, como se vê ao abrir uma garrafa de champanhe ou refrigerante, onde bolhas de $\mathrm{CO}_{2}$ se desprendem da fase líquida.

A constante de transferência pode ser determinada experimentalmente ao observar a concentração do composto $C$ em função do tempo em uma solução sub ou supersaturada. Pela integração da Eq. 1, tem-se o caso de uma concentração uniforme de $\mathrm{C}$ na fase líquida:

$\operatorname{Ln}\left(\left(C_{s}-C\right) /\left(C_{s}-C_{o}\right)\right)=-k_{d} t$

Em que:

$\mathrm{C}_{\mathrm{o}}=$ concentração inicial de C (mg/L);

$\mathrm{t}=$ tempo $(\mathrm{h})$

Aplicando a Eq. 2 para os componentes voláteis $\mathrm{OD}, \mathrm{CO}_{2}$ e $\mathrm{NH}_{3}$ tem-se:

$$
\begin{aligned}
& \operatorname{Ln}\left(\left([\mathrm{OD}]_{\mathrm{s}}-[\mathrm{OD}]\right) /\left([\mathrm{OD}]_{\mathrm{s}}-[\mathrm{OD}]_{\mathrm{o}}\right)\right)=-\mathrm{k}_{\mathrm{do}} \mathrm{t} \\
& \operatorname{Ln}\left(\left(\left[\mathrm{CO}_{2}\right]_{\mathrm{s}}-\left[\mathrm{CO}_{2}\right]\right) /\left(\left[\mathrm{CO}_{2}\right]_{\mathrm{s}}-\left[\mathrm{CO}_{2}\right]_{\mathrm{o}}\right)\right)=-\mathrm{k}_{\mathrm{dc}} \mathrm{t} \\
& \operatorname{Ln}\left(\left[\mathrm{NH}_{3}\right] /\left[\mathrm{NH}_{3}\right]_{\mathrm{o}}\right)=-\mathrm{k}_{\mathrm{dn}} \mathrm{t}
\end{aligned}
$$

Em que:

$k_{d o}, k_{d c}$ e $k_{d n}=$ constantes de transferência de OD, $\mathrm{CO}_{2}$ e $\mathrm{NH}_{3}$, respectivamente

Índice "s" e "o" indicam valores de saturação e iniciais

Como absorção e dessorção são fenômenos que ocorrem na superfície da fase líquida e, para um determinado volume, a área é inversamente proporcional à profundidade da fase líquida, se a área não varia com a profundidade. Pode-se então esperar que o valor das constantes seja inversamente proporcional à profundidade:

$\mathrm{k}_{\mathrm{dh}}=\mathrm{k}_{\mathrm{d} 1} / \mathrm{H}$

Em que:

$\mathrm{k}_{\mathrm{dh}}=$ valor $\mathrm{da}$ constante a uma profundidade qualquer $\mathrm{H}$

$\mathrm{k}_{\mathrm{d} 1}=$ valor da constante a uma profundidade $\mathrm{H}=1 \mathrm{~m}$ $\mathrm{H}=$ profundidade da fase líquida $(\mathrm{m})$ 
A constante também depende da temperatura. Supondo uma dependência tipo Arrehnius, que tem sido observada por vários pesquisadores (KLOCK, 1971), tem-se:

$k_{d T}=k_{d 20} \theta^{(T-20)}$

Em que:

$k_{d T}=$ valor da constante a uma temperatura qualquer

$\mathrm{k}_{\mathrm{d} 20}=$ valor da constante a uma temperatura padrão de $20^{\circ} \mathrm{C}$

$\mathrm{T}=$ temperatura da fase líquida $\left({ }^{\circ} \mathrm{C}\right)$

$\theta=$ coeficiente de dependência da temperatura

Assim, tem-se para qualquer profundidade e temperatura que:

$k_{d T h}=\left(k_{d 20,1} / H\right) \theta^{(T-20)}$

Em que:

$\mathrm{k}_{\mathrm{dTh}}=$ constante de transferência para uma determinada temperatura e profundidade

$\mathrm{k}_{\mathrm{d} 20,} 1=$ constante de transferência para condições padrão $\left(t=20^{\circ} \mathrm{C} ; \mathrm{H}=1 \mathrm{~m}\right)$

Portanto, para saber o valor da constante de transferência de um componente volátil para qualquer profundidade é necessário saber seu valor para um determinado valor da temperatura e da profundidade e a dependência do valor da constante da temperatura e da profundidade.

\section{METODOLOGIA}

Realizou-se uma investigação experimental para determinar a influência da profundidade e da temperatura do líquido em lagoas sobre o valor das constantes de transferência dos três componentes voláteis $\left(\mathrm{O}_{2}, \mathrm{CO}_{2}, \mathrm{NH}_{3}\right)$. Os ensaios foram realizados em escala piloto utilizando protótipos de lagoas com 0,2 e 0,4 m de profundidade e diâmetro de $0,5 \mathrm{~m}$ (volumes de $40 \mathrm{e}$ $80 \mathrm{~L}$, respectivamente).

$\mathrm{Na}$ primeira fase da investigação, usaram-se concentrações dos compostos voláteis na faixa de valores normalmente encontrados no afluente de lagoas de polimento. Numa segunda fase usaram-se valores maiores dos compostos para estabelecer a que concentração a supersaturação levava à formação de bolhas de gás que se desprendem.

A Fig. 1 mostra uma representação esquemática do experimento montado para determinar o valor das constantes, na primeira fase, e a concentração mínima para formação de bolhas se desprendendo na segunda fase. Colocou-se água em modelos de lagoas com 0,20 (L1) e 0,40 (L2) $\mathrm{m}$ de profundidade, mantendo-se temperaturas controladas de 20 e $30^{\circ} \mathrm{C}$ para o oxigênio dissolvido e o dióxido de carbono e de 20 e $27,5^{\circ} \mathrm{C}$ para a amônia.

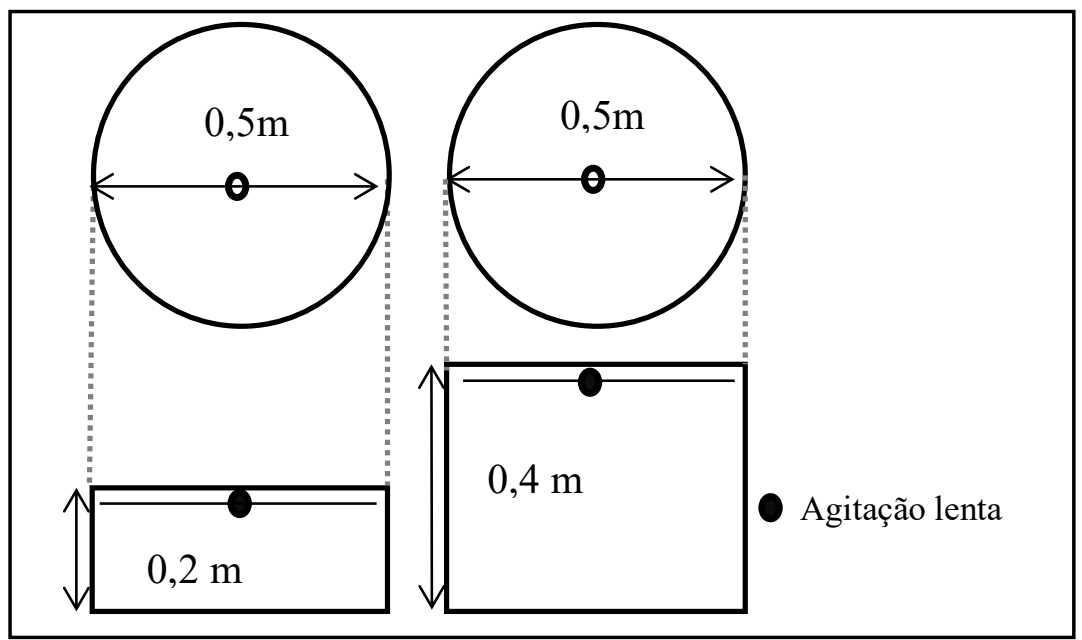

Figura 1 - Representação esquemática das lagoas utilizadas nos ensaios de determinação das constantes. 
Dependendo da finalidade do teste, adicionavamse à água concentrações conhecidas de um dos três componentes voláteis $\left(\mathrm{O}_{2}, \mathrm{CO}_{2}, \mathrm{NH}_{3}\right)$ e, após eventual correção do $\mathrm{pH}$, era observada a variação da concentração dos componentes voláteis em função do tempo, o que permitia calcular o valor das constantes. Para determinar o valor das constantes de transferência, convém realizar experimentos em reatores operados em regime de batelada, onde a concentração do componente volátil varia com o tempo da maneira prevista nas Eq. 2a, 2b e 2c.

\subsection{Constante de transferência de oxigênio dissolvido, $\mathrm{k}_{\mathrm{do}}$}

Para determinar a constante de absorção de OD, eliminou-se o OD da água mediante a adição de sulfito de sódio, tendo-se adicionado antes 50 $\mathrm{mg} / \mathrm{L}$ de cloreto de cobalto $\left(\mathrm{CoCl}_{2}\right)$, que catalisa a oxidação de sulfito pelo OD (VAN HAANDEL; MARAIS, 1999). Observou-se então o valor da concentração de OD nas lagoas, enquanto se aplicava suave agitação para uniformizar a concentração de OD. A concentração de OD foi monitorada com auxílio de um medidor multiparâmetro (Hanna HI $98196 \mathrm{pH}$ /ORP/DO), com dados registrados a cada 10 minutos. De posse dos dados, utilizou-se então a Eq. 2a para calcular o valor da constante nas lagoas. A concentração de saturação de oxigênio para os experimentos, considerando 550 metros acima do nível do mar, foi 8,5 e 7,1 mg/L, para as temperaturas de 20 e $30^{\circ} \mathrm{C}$, respectivamente.

O efeito da agitação foi avaliado na constante de absorção; assim, da mesma forma que se procedeu para determinação da constante de absorção com agitação, seguiu-se para as lagoas sem agitação.

O experimento era repetido com uma solução supersaturada de oxigênio criado com peróxido de hidrogênio que era decomposto em água e oxigênio nos tanques por adição de óxido de manganês, criando-se, assim, uma solução com uma alta concentração de oxigênio dissolvido.

\subsection{Constante de transferência de dióxido de carbono, $\mathrm{k}_{\mathrm{dc}}$}

Para determinar a constante de transferência de $\mathrm{CO}_{2}$, preparou-se uma solução supersaturada do gás na água nos modelos de lagoas, dissolvendo $20 \mathrm{mmol} / \mathrm{L}$ de $\mathrm{NaHCO}_{3}$ e baixando o $\mathrm{pH}$ para aproximadamente 5,5 , transformando grande parte do bicarbonato em $\mathrm{CO}_{2}$ e criando-se uma solução supersaturada deste gás. Os valores de $\mathrm{pH}$ e temperatura foram monitorados com auxílio do medidor multiparâmetro (Hanna HI 98196 pH/ORP/DO), sendo registrados a cada hora, e a alcalinidade foi determinada segundo metodologia descrita por Buchauer (1998).

De posse dos dados experimentais, calculou-se a constante de transferência de $\mathrm{CO}_{2}$ adotando-se o seguinte procedimento:

(1) Loewenthal e Marais (1976) definiram a alcalinidade e a acidez de uma água como:

$$
\begin{aligned}
& \text { Alc }=2\left[\mathrm{CO}_{3}=\right]+\left[\mathrm{HCO}_{3}^{-}\right]+\left[\mathrm{OH}^{-}\right]-\left[\mathrm{H}^{+}\right] \\
& \mathrm{Ac}=2\left[\mathrm{CO}_{2}\right]+\left[\mathrm{HCO}_{3}^{-}\right]+\left[\mathrm{H}^{+}\right]-\left[\mathrm{OH}^{-}\right]
\end{aligned}
$$

$\mathrm{Na}$ faixa de $\mathrm{pH}=5$ a $\mathrm{pH}=9$ (que foi a faixa utilizada no experimento), os valores das concentrações de $\mathrm{H}+$ e $\mathrm{OH}$ - são muito pequenas e em boa aproximação tem-se:

$$
\begin{aligned}
& \text { Alc }=2\left[\mathrm{CO}_{3}{ }^{-}\right]+\left[\mathrm{HCO}_{3}^{-}\right] \\
& \mathrm{AC}=2\left[\mathrm{CO}_{2}\right]+\left[\mathrm{HCO}_{3}^{-}\right]=\left[\mathrm{CO}_{2}\right]\left(2+\mathrm{K}_{1} /\left[\mathrm{H}^{+}\right]\right)
\end{aligned}
$$

(2) As Eq. 4a e 5a podem ser reescritas como a Equação de Deffeyes:

$\mathrm{Alc} \approx \mathrm{Ac}\left[1+2,10^{(\mathrm{pH}-\mathrm{pk} 2)}\right] /\left[1+2,10^{(\mathrm{pk} 1-\mathrm{pH})}\right] \quad(5<\mathrm{pH}<9)(6)$

(3) Para diferentes valores do $\mathrm{pH}$ e o valor médio da alcalinidade medidos em uma batelada de água com $\mathrm{CO}_{2}$ supersaturado, calcula-se a acidez com a Eq. 6;

(4) Calcula-se a concentração de $\mathrm{CO}_{2}: \mathrm{CO}_{2}=\mathrm{Acl}$ $\left(2+10^{(\mathrm{pH}-\mathrm{pk} 1)}\right)$; 
(5) Plota-se $\ln \left[\left(\mathrm{CO}_{2, t}-\mathrm{CO}_{2, t+1}\right) /\left(\mathrm{CO}_{2,0}-\mathrm{CO}_{2 s}\right)\right]$ em função do tempo de transferência;

(6) Estabelece-se a declividade da reta que meIhor descreve a tendência que é o valor da constante de transferência.

Os experimentos eram realizados com duas concentrações iniciais de $\mathrm{CO}_{2}$, sendo um com um valor compatível com a concentração de $\mathrm{CO}_{2}$ no efluente de um UASB tratando esgoto $\left(\mathrm{CO}_{2}=5 \mathrm{meq} / \mathrm{L}\right)$ e outro com uma concentração 10 vezes maior para avaliar a possibilidade de formação de bolhas de $\mathrm{CO}_{2}$ na fase líquida.

\subsection{Constante de transferência de amônia, kdn}

A solução supersaturada de $\mathrm{NH}_{3}$ era preparada da seguinte maneira: aumentava-se o $\mathrm{pH}$ da água até um valor de 12 aproximadamente usando hidróxido de sódio, o que resultava na precipitação de carbonato de cálcio. Depois de deixar a água em repouso por 24 horas, separava-se o sobrenadante e adicionavam-se $100 \mathrm{mgN} / \mathrm{L}$ na forma de bicarbonato de amônia e transferia-se essa solução para os tanques de transferência.

Os valores da constante de transferência eram obtidos conforme o seguinte roteiro:

(1) Para as diferentes profundidades, determinar o pH, a concentração de amônio total $\left(\mathrm{N}_{\text {tot }}=\mathrm{NH}_{4}{ }^{+}\right.$ $+\mathrm{NH}_{3}$ ) e a alcalinidade em função do tempo e calcular a concentração de amônia não ionizada $\left(\mathrm{NH}_{3}\right)$.
(2) Calcular, para cada intervalo de tempo, a razão $\Delta \mathrm{NH}_{3} / \Delta \mathrm{t}=\left(\mathrm{NH}_{3, i+1}-\mathrm{NH}_{3} \mathrm{i}\right) /\left(\mathrm{t}_{\mathrm{i}+1}-\mathrm{t}_{\mathrm{i}}\right)$.

(3) Calcular os valores de $k_{d n}$ para os diferentes intervalos com auxílio da Eq. 2c.

(4) Determinar a constante para diferentes profundidades e temperaturas.

(5) Verificar se a diminuição observada da alcalinidade é compatível com a diminuição de $\left[\mathrm{NH}_{3}\right]+\left[\mathrm{NH}_{4}^{+}\right]$.

Novamente realizaram-se dois experimentos, sendo um com uma concentração inicial de amônia com um valor compatível com a concentração de $\mathrm{CO}_{2}$ no efluente de um UASB tratando esgoto (amônia $\approx 4 \mathrm{meq} / \mathrm{L}$ ) e outro com uma concentração 5 vezes maior para avaliar a possibilidade de formação de bolhas de $\mathrm{NH}_{3}$ na fase líquida.

\section{RESULTADOS}

\subsection{Constante de Transferência de $\mathrm{O}_{2}$}

$\mathrm{Na}$ Fig. 2, pode-se observar o gráfico de $\left.\operatorname{Ln}\left\{\left[(O D)_{s} \text { - (OD)]/[(OD) }\right)_{s}(O D)_{o}\right]\right\}$ em função do tempo para profundidades de 0,2 e 0,4 $\mathrm{m}$ e temperaturas controladas de 20 e $30^{\circ} \mathrm{C}$. Nota-se que em todos os casos os valores experimentais plotam sobre uma reta, cuja declividade é igual à constante de transferência (Eq. 2a). Portanto, foi observado que a lei de Fick se aplica em todos os casos.
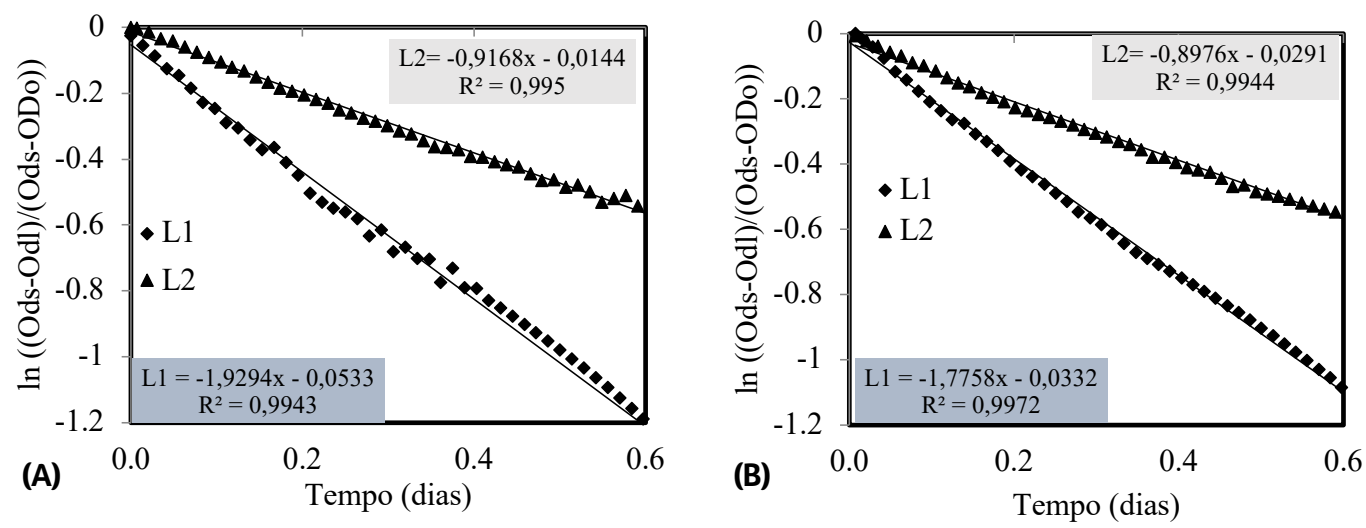

Figura 2 - Experimento para determinar a constante de absorção de oxigênio a $20^{\circ} \mathrm{C}(\mathrm{A})$ e a $30^{\circ} \mathrm{C}(\mathrm{B})$ para as duas profundidades. 

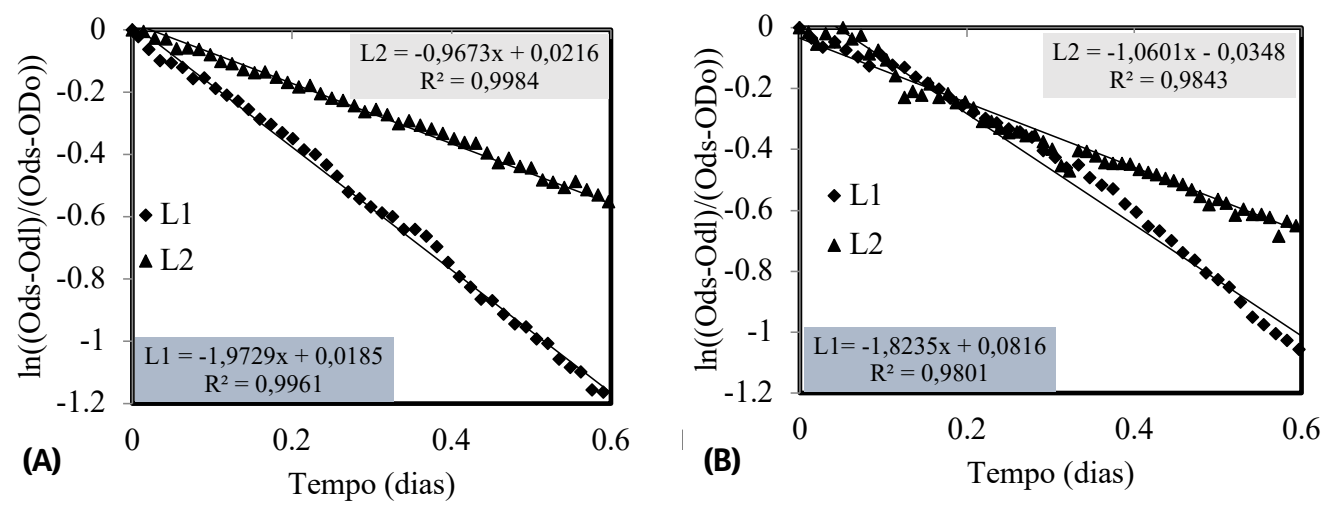

Figura 3 - Experimento de transferência a $20^{\circ} \mathrm{C}(\mathrm{A})$ e a $30^{\circ} \mathrm{C}(\mathrm{B})$ para as duas profundidades.

Com os resultados do experimento de OD, foi possível traçar retas que permitiram estabelecer os valores para as diferentes profundidades e temperaturas. Com esses valores e as Eq. 3 a e 3b, pode-se derivar a expressão geral para a absorção de OD em lagoas:

OD subsaturado: $k_{\text {doTh }}=1,001^{(T-20) * 0,374 / H}$

O experimento foi repetido com soluções supersaturadas de OD, da mesma forma que se procedeu com o experimento subsaturado. Os resultados estão representados na Fig. 3. Verifica-se que em todos os casos novamente os valores experimentais plotam sobre uma reta com declividade igual à constante de transferência (Eq. 2a). A variação também pôde ser descrita com a lei de Fick, mas nesse caso o valor da constante determinada foi um pouco diferente:

OD supersaturado: $k_{\text {doTh }}=1,001^{(T-20) * 0,391 / H \quad(8)}$

Assim, a melhor estimativa para a constante de transferência de oxigênio dissolvido em lagoas é dada por:

$k_{\text {doTh }}=1,001^{(T-20)} 0,382 / H$

\subsection{Constante de Transferência de $\mathrm{CO}_{2}$}

A constante de transferência de dióxido de carbono obtida foi alcançada usando-se soluções supersaturadas do gás em tanques de água com profundidades de 0,2 e 0,4 m. Novamente, estabeleceu-se a validade da lei de Fick pela Eq. $2 b$. Usando-se os dados experimentais foram obtidas as retas como mostrado na Fig. 4 e calculouse a constante de transferência em função da profundidade e da temperatura (Eq. 10):

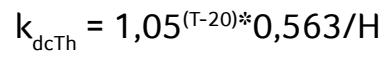
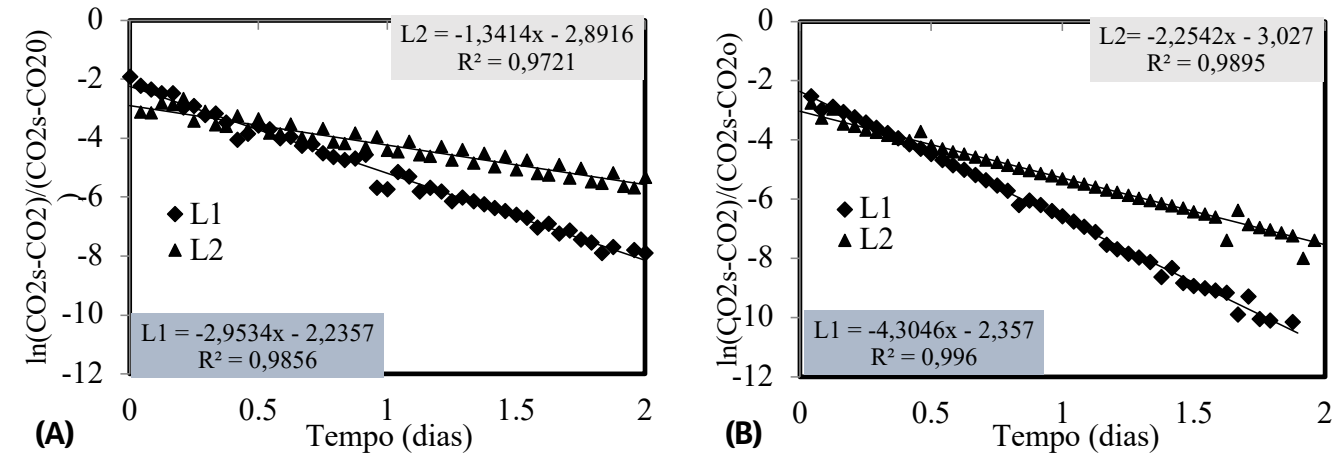

Figura 4 - Experimento de transferência de $\mathrm{CO}_{2}$ a $20^{\circ} \mathrm{C}(\mathrm{A})$, e a $30^{\circ} \mathrm{C}(\mathrm{B})$ para as duas profundidades de lagoa investigadas. 


\subsection{Constante de Transferência de $\mathrm{NH}_{3}$}

Nas Tabelas 1 e 2 estão dispostos os resultados obtidos para determinação da constante de transferência de $\mathrm{NH} 3$ na temperatura de $20^{\circ} \mathrm{C}$ nas duas lagoas, com profundidades de 0,2 e 0,4 m.

Tabela 1 - Valores das constantes de transferência de $\mathrm{NH}_{3}$ obtidos a partir dos valores do $\mathrm{pH}$, concentração de amônia total e amônia na Lagoa de $0,2 \mathrm{~m}$ na temperatura $1\left(20^{\circ} \mathrm{C}\right)$.

\begin{tabular}{|c|c|c|c|c|c|c|}
\hline Tempo (dias) & $\mathrm{pH}$ & Alc (meq/L) & $\mathrm{NH}_{3}$ tot $(\mathrm{mg} / \mathrm{L})$ & $\mathrm{NH}_{3}(\mathrm{mg} / \mathrm{L})$ & rdN & $\mathrm{K}_{\mathrm{NH3}}$ \\
\hline 0 & 9,26 & 8,26 & 54,0 & 22,4 & - & - \\
\hline 1 & 8,80 & 8,10 & 40,9 & 8,1 & 13,05 & 1,607 \\
\hline 2 & 8,62 & 7,30 & 36,9 & 5,1 & 4,03 & 0,778 \\
\hline 3 & 8,54 & 7,09 & 32,4 & 3,8 & 4,48 & 1,154 \\
\hline 4 & 8,49 & 6,76 & 29,1 & 3,1 & 3,36 & 1,069 \\
\hline 5 & 8,44 & 6,23 & 26,9 & 2,6 & 2,13 & 0,810 \\
\hline 6 & 8,39 & 5,85 & 25,2 & 2,2 & 1,79 & 0,811 \\
\hline 7 & 8,35 & 5,59 & 23,5 & 1,9 & 1,68 & 0,886 \\
\hline
\end{tabular}

Tabela 2 - Valores das constantes de transferência de $\mathrm{NH}_{3}$ obtidos a partir dos valores do pH, concentração de amônia total e amônia na Lagoa de $0,4 \mathrm{~m}$ na temperatura $1\left(20^{\circ} \mathrm{C}\right)$.

\begin{tabular}{|c|c|c|c|c|c|c|}
\hline Tempo (dias) & $\mathbf{p H}$ & $\mathbf{A l c} \mathbf{( m e q} / \mathbf{L})$ & $\left.\mathbf{N H}_{3} \mathbf{t o t} \mathbf{( m g} / \mathbf{L}\right)$ & $\mathbf{N H}_{\mathbf{3}} \mathbf{( m g} \mathbf{L} \mathbf{)}$ & $\mathbf{r d N}$ & $\mathbf{K}_{\mathbf{N H 3}}$ \\
\hline 0 & 9,5 & 8,5 & 54,1 & 29,9 & - & - \\
\hline 1 & 9,47 & 7,5 & 43,6 & 23,4 & 10,47 & 0,447 \\
\hline 2 & 9,35 & 6,7 & 38,6 & 18,0 & 5,04 & 0,279 \\
\hline 3 & 9,23 & 6,5 & 34,7 & 13,8 & 3,92 & 0,283 \\
\hline 4 & 9,14 & 6 & 30,2 & 10,6 & 4,48 & 0,422 \\
\hline 5 & 9,05 & 5,8 & 27,4 & 8,3 & 2,80 & 0,334 \\
\hline 6 & 8,96 & 5,6 & 22,4 & 5,8 & 5,04 & 0,855 \\
\hline 7 & 8,9 & 5,5 & 19,0 & 4,5 & 3,36 & 0,744 \\
\hline
\end{tabular}

Nas Tabelas 3 e 4 estão dispostos os resultados obtidos para determinação da constante de transferência de NH3 na temperatura $27,5^{\circ} \mathrm{C}$ nas duas lagoas com profundidades de, 0,2 e 0,4 m.

Tabela 3 - Valores das constantes de transferência de $\mathrm{NH}_{3}$ obtidos a partir dos valores do pH, concentração de amônia total e amônia na Lagoa de $0,2 \mathrm{~m}$ na temperatura $2\left(27,5^{\circ} \mathrm{C}\right)$.

\begin{tabular}{|c|c|c|c|c|c|c|}
\hline Tempo (dias) & $\mathrm{pH}$ & Alc (meq/L) & $\mathrm{NH}_{3}$ tot $(\mathrm{mg} / \mathrm{L})$ & $\mathrm{NH}_{3}(\mathrm{mg} / \mathrm{L})$ & $\mathrm{rdN}$ & $\mathbf{K}_{\mathrm{NH} 3}$ \\
\hline 0 & 9,54 & 9,2 & 55,8 & 38,9 & & \\
\hline 1 & 9,22 & 7,5 & 33,3 & 17,5 & 22,48 & 1,283 \\
\hline 2 & 9,04 & 6,9 & 18,7 & 7,9 & 14,60 & 1,845 \\
\hline 3 & 8,8 & 6,4 & 11,6 & 3,4 & 7,11 & 2,068 \\
\hline 4 & 8,7 & 6,3 & 7,2 & 1,8 & 4,37 & 2,409 \\
\hline 5 & 8,6 & 6,2 & 5,1 & 1,0 & 2,12 & 1,981 \\
\hline
\end{tabular}

Tabela 4 - Valores das constantes de transferência de $\mathrm{NH}_{3}$ obtidos a partir dos valores do pH, concentração de amônia total e amônia na Lagoa de $0,4 \mathrm{~m}$ na temperatura $2\left(27,5^{\circ} \mathrm{C}\right)$.

\begin{tabular}{|c|c|c|c|c|c|c|}
\hline Tempo (dias) & $\mathrm{pH}$ & Alc (meq/L) & $\mathrm{NH}_{3}$ tot $(\mathrm{mg} / \mathrm{L})$ & $\mathrm{NH}_{3}(\mathrm{mg} / \mathrm{L})$ & $\mathrm{rdN}$ & $\mathbf{K}_{\mathrm{NH} 3}$ \\
\hline 0 & 9,5 & 9,5 & 54,9 & 37,3 & & \\
\hline 1 & 9,30 & 8,5 & 36,9 & 21,1 & 18,03 & 0,854 \\
\hline 2 & 9,18 & 7,7 & 27,3 & 13,7 & 9,63 & 0,701 \\
\hline 3 & 9,11 & 7,5 & 19,5 & 9,0 & 7,83 & 0,867 \\
\hline 4 & 9,03 & 7 & 13,4 & 5,5 & 6,10 & 1,091 \\
\hline 5 & 8,99 & 6,8 & 8,0 & 3,1 & 5,40 & 1,708 \\
\hline
\end{tabular}


Os resultados dos experimentos mostram uma relação inversamente proporcional da constante de transferência de $\mathrm{NH}_{3}$ com a profundidade; tal fato também foi reportado por Cavalcanti (2009). A partir dos resultados obtidos na temperatura padrão de $20^{\circ} \mathrm{C}$ para as duas profundidades analisadas, podese extrapolar o valor da constante para uma profundidade de $1 \mathrm{~m}$ obtendo-se um valor aproximado da constante de transferência de $0,20 \mathrm{~d}^{-1}$. Por meio da correlação entre os resultados experimentais da constante de transferência com as duas profundidades analisadas e utilizando a Eq. 3a observa-se a influência da profundidade como (Eq. 11):

$K_{d n}=\frac{0,198}{H}$

Para determinar o efeito da temperatura no valor da constante a partir da equação 3(b), foi constatado que, no intervalo de temperatura investigado, a constante praticamente dobrou. Assim, a expressão para a constante de transferência de $\mathrm{NH}_{3}$ pode ser expressa em função da temperatura ( $\mathrm{t}$ em Celsius) e da profundidade ( $\mathrm{H}$ em metros) por meio da equação 3(c) da seguinte forma:

$K_{d n T h}=\frac{0,198 * 1,095^{(T-20)}}{H}$

Os dados obtidos mostram com boa aproximação que as constantes de absorção e transferência são inversamente proporcionais à profundidade, ou seja, quanto menor a profundidade, maior será a velocidade de transferência e/ou absorção. Com o aumento da temperatura, dadas praticamente as mesmas condições iniciais para o teste de transferência de amônia, foi perceptível que em um tempo bem menor (dois e três dias, respectivamente para as lagoas de 0,2 e 0,4m) obtiveram-se valores de amônia total inferiores ao teste a $20^{\circ} \mathrm{C}$ nas mesmas profundidades, o que explicitamente se refletiu na constante, que teve seu valor duplicado.

\subsection{Determinação da faixa de validade da Lei de Fick}

Realizaram-se testes com soluções supersaturadas dos três componentes voláteis. Na transferência do OD, iniciou-se com uma concentração de aproximadamente $50 \mathrm{mg} / \mathrm{L}$, e foi possível verificar que houve uma rápida transferência em decorrência do grande desprendimento de bolhas de oxigênio. Observou-se que a lei de Fick é obedecida quando a concentração de OD se torna inferior a $35 \mathrm{mg} / \mathrm{L}$, como pode ser observado na Fig. 5. Na Fig. 5A plotou-se a concentração medida de OD em função do tempo. Na Fig. 5B plotou-se o valor de $\operatorname{Ln}\left(\left([O D]_{s}-[O D]\right) /\left([O D]_{s}-[O D]_{o}\right)\right)$ em função do tempo. Os dados só plotam numa reta para $[O D]_{0}<35 \mathrm{mg} / \mathrm{L}$, o que representa o limite da validade da lei de Fick. Verifica-se que a parte linear da Fig. 5B apresenta grande similaridade com a obtida na Fig. 2 (A e B). Na prática, a concentração de OD em lagoas de polimento frequentemente pode ficar acima da concentração de saturação (7-8 mg/L), mas dificilmente chega a um valor de $35 \mathrm{mg} / \mathrm{L}$. Portanto, pode-se afirmar que a transferência de oxigênio em lagoas de tratamento se dá de acordo com a lei de Fick.

Para a transferência de $\mathrm{CO}_{2}$, iniciou-se o teste com uma concentração de aproximadamente 80 meq/L. Os resultados estão plotados na Fig. 6 . Pode-se observar que, inicialmente, a transferência de $\mathrm{CO}_{2}$ ocorre aceleradamente, mas quando a concentração alcança $40 \mathrm{meq} / \mathrm{L}$ começa a se comportar de acordo com a lei de Fick. A declividade encontrada na Fig. 6B ( $L 1=-4,2417$ e $L 2=-2,4712$ ) apresenta grande similaridade à encontrada na Fig. 4B quando da obtenção da constante de dessorção de $\mathrm{CO}_{2}$ para a mesma temperatura. Pode-se estimar a concentração de $\mathrm{CO}_{2}$ no efluente de um UASB a partir de valores "normais" de $\mathrm{pH}$ (7) e alcalinidade (8 meq/L). Nessas condições, a concentração de $\mathrm{CO}_{2}$ é menor que $10 \mathrm{meq} / \mathrm{L}$. Conclui-se que para lagoas de tratamento a transferência de $\mathrm{CO}_{2}$ é descrita pela 
Lei de Fick. Como o valor da constante de $\mathrm{CO}_{2}$ tem um valor relativamente elevado, a dessorção de $\mathrm{CO}_{2}$ no efluente de um UASB normalmente se dá em pouco tempo: 1 a 2 dias. Quando o $\mathrm{pH}$ aumenta e atinge valores acima de 8,5-9, a absorção de $\mathrm{CO}_{2}$ ocorre.
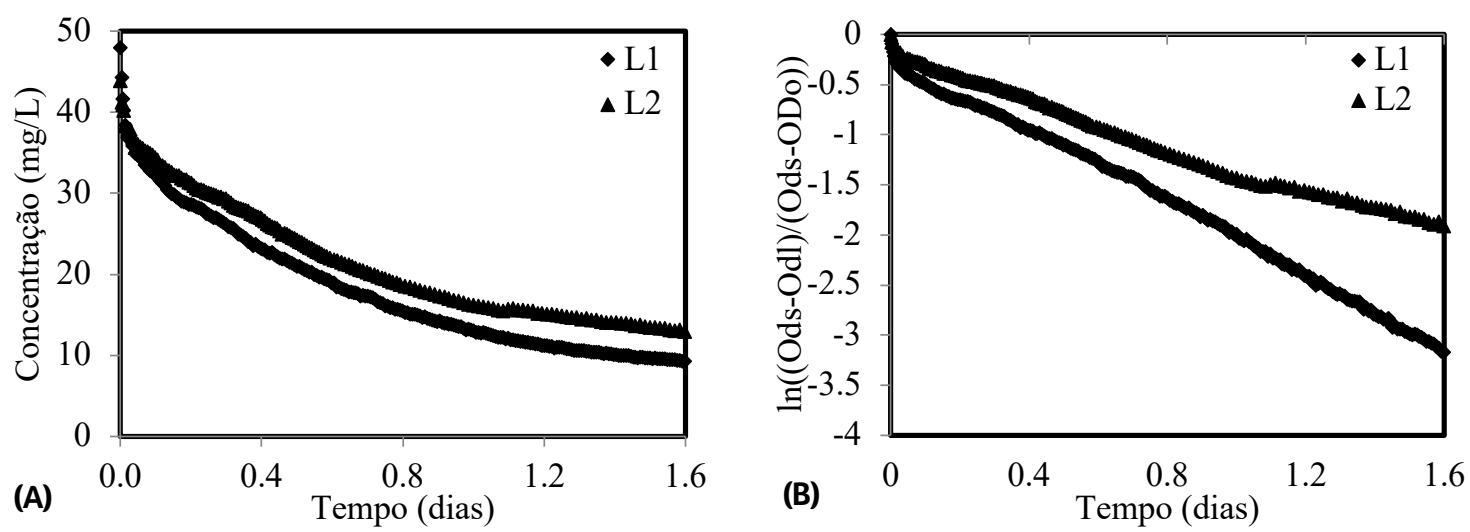

Figura 5 - Experimento de supersaturação de oxigênio nas duas profundidades de lagoa investigadas (A) concentração de OD e (B) influência dos pontos na determinação da reta.
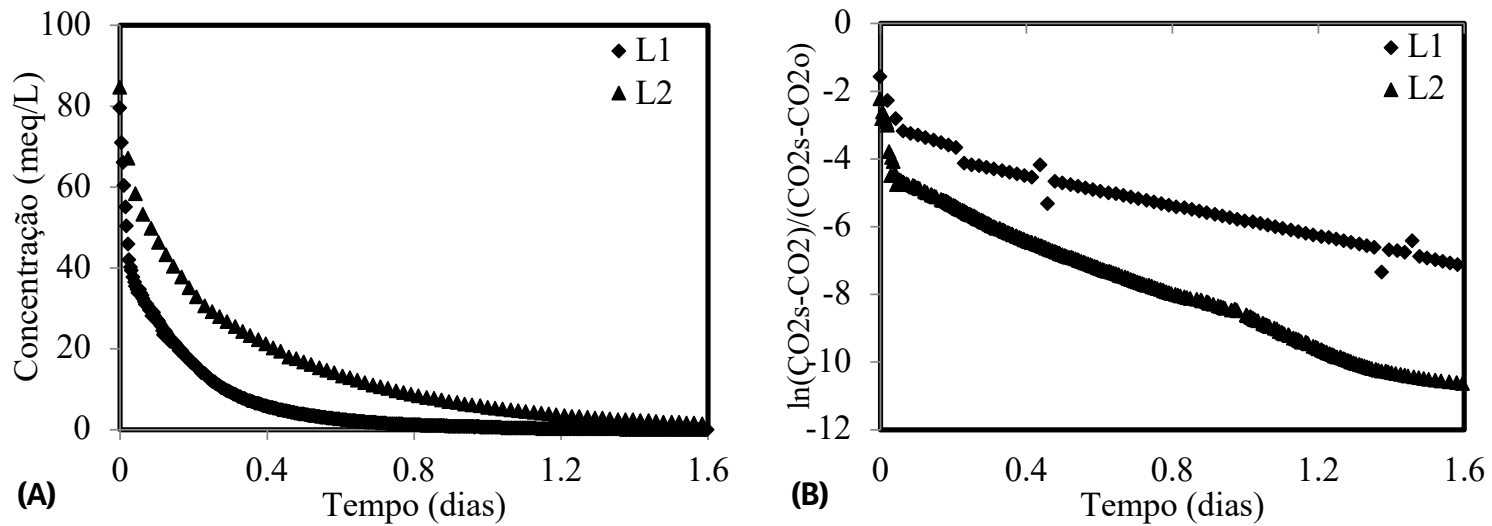

Figura 6 - Experimento de supersaturação de dióxido de carbono nas duas profundidades de lagoa investigadas em (A) concentração de $\mathrm{CO}_{2}$ e (B) influência dos pontos na determinação da reta.

Para a transferência de amônia observa-se na Fig. 7A que inicialmente ocorre uma rápida transferência, mas quando a concentração se torna menor que $60 \mathrm{mg} / \mathrm{L}$ essa taxa começa a se apresentar de acordo com a lei de Fick, conforme se constata na Fig. 7B. Ademais, a declividade da parte linear da Fig. 7B é similar às constantes de transferência obtidas nas Tabelas 1 e 2, para a mesma temperatura $\left(20^{\circ} \mathrm{C}\right)$. Dessa forma, po- de-se inferir que a lei de Fick é válida quando a concentração de amônia está abaixo de $60 \mathrm{mg} / \mathrm{L}$, o que na prática quase sempre se verifica para o tratamento de esgoto.

Assim, conclui-se que quase sempre em lagoas de polimento a dessorção dos compostos voláteis $\mathrm{OD}, \mathrm{CO}_{2}$ e $\mathrm{NH}_{3}$ ocorre de acordo com a lei de Fick. 

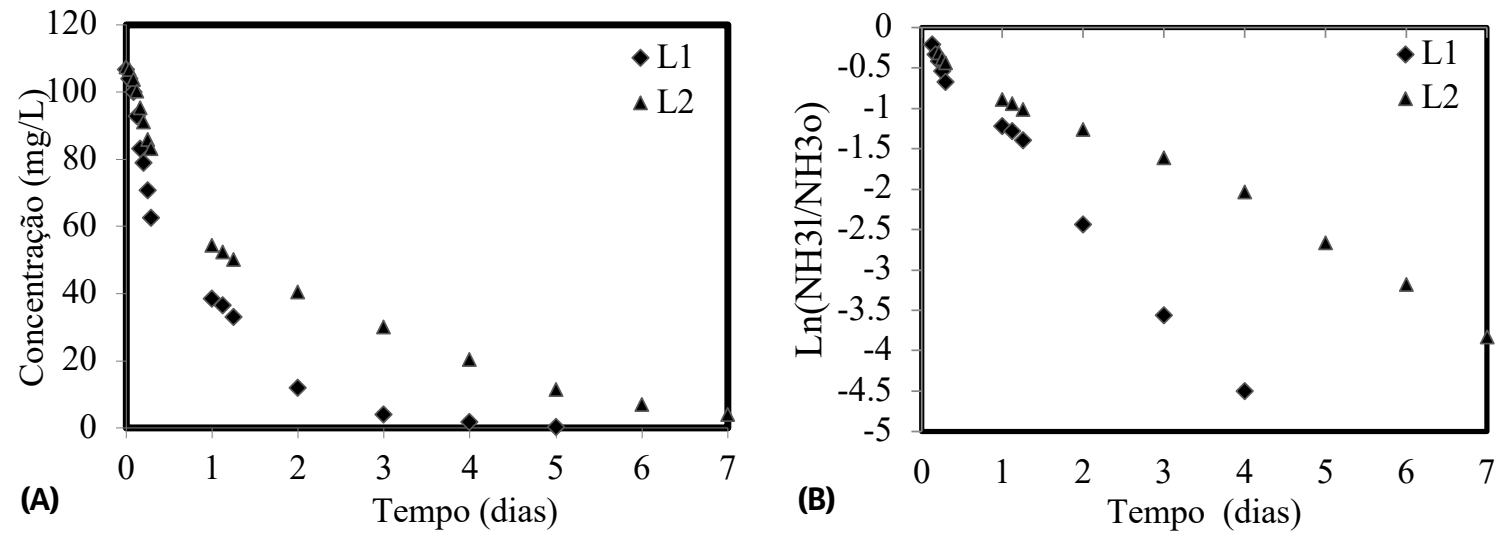

Figura 7 - Experimento de supersaturação de amônia nas duas profundidades de lagoa investigadas em (A) concentração de amônia e (B) influência dos pontos na determinação da reta.

\section{DISCUSSÃO}

$\mathrm{O}$ aspecto divergente mais importante de lagoas de polimento de bateladas sequenciais (LPBS) e de fluxo contínuo (LPFC) é que nas LPBS a demanda de oxigênio para oxidação varia com o tempo, sendo maior logo depois que se inicia o tratamento de uma batelada. Como inicialmente não há algas na LPBS, a demanda tem de ser atendida pela absorção de oxigênio atmosférico. Se a concentração de OD inicialmente é zero, a taxa de transferência é dada pela Eq. 13.

$r_{d o}=k_{d o} O D_{s}=1,001^{(T-20)} 0,38 / H^{*}(51,6 / 31,6+T) * 9,2$

Em que:

$\mathrm{r}_{\mathrm{do}}=(\mathrm{dC} / \mathrm{dt})_{\mathrm{a}}=$ taxa de transferênica de OD $(\mathrm{mg} / \mathrm{L} / \mathrm{h})$;

$\mathrm{k}_{\mathrm{do}}=$ constantes de transferência de OD $\left(\mathrm{h}^{-1}\right)$;

$\mathrm{T}=$ temperatura $\left({ }^{\circ} \mathrm{C}\right)$;

$\mathrm{H}=$ profundidade $(\mathrm{m})$.

Assim, por exemplo, para uma profundidade de $0,4 \mathrm{~m}$ e uma temperatura de $25^{\circ} \mathrm{C}$, a taxa de absorção é calculada em $8 \mathrm{mg} \cdot \mathrm{L}^{-1} \cdot \mathrm{d}^{-1}$. Esse valor é alto comparado com o valor da demanda de oxigênio. A demanda de oxigênio no primeiro dia da batelada pode ser estimada a partir da equação de Phelps (1944), que prevê uma diminuição exponencial da DBO, conforme a Eq. 14.

$D B O_{t}=D B O_{i} \exp \left(-k_{p h} t\right)$
Em que:

$\mathrm{DBO}_{\mathrm{t}}, \mathrm{DBO}_{\mathrm{i}}=\mathrm{DBO}$ depois de um tempo t e DBO inicial respectivamente $(\mathrm{mg} / \mathrm{L})$

$\mathrm{K}_{\mathrm{ph}}=$ constante de Phelps $=0,23 \mathrm{~d}^{-1}$

$\mathrm{t}=$ tempo desde $\mathrm{o}$ início da batelada $(\mathrm{d})$

Tendo-se, por exemplo, uma DBO de $50 \mathrm{mg} \cdot \mathrm{L}^{-1}$ no efluente do reator UASB, calcula-se a DBO depois de $1 \mathrm{~d}$ como DBO1 = 39,7 mg.L-1 , de modo que a demanda no primeiro dia é estimada em 50-39,7 = 10,3 mg. $\mathrm{L}^{-1}$. Portanto, a transferência de oxigênio ( $\left.8 \mathrm{mg} \cdot \mathrm{L}^{-1} \mathrm{~d}^{-1}\right)$ é da mesma ordem de grandeza da demanda de oxigênio. Nessas condições, depois do primeiro dia da operação da batelada a concentração de OD continuaria zero, mas a partir do segundo dia haveria oxigênio dissolvido, não somente por causa da absorção de oxigênio, mas principalmente porque a partir do segundo dia já haveria presença de algas na lagoa e consequentemente produção fotossintética de OD. Se a DBO do efluente do UASB for maior que $50 \mathrm{mg} \cdot \mathrm{L}^{-1}$ ou se a profundidade da lagoa for maior que o 0,4 $\mathrm{m}$ que foi pressuposto, o tempo para aparecer OD na LPBS será mais longo, mas invariavelmente depois de um período inicial, OD aumenta com o tempo. Conclui-se que a absorção de OD por LPBS somente é importante nos primeiros dias de operação: depois 
a produção de oxigênio fotossintético predomina e o ambiente se torna aeróbio.

O mecanismo de dessorção predomina na remoção de amônia. Os processos alternativos de assimilação na biomassa e nitrificação-desnitrificação praticamente não se desenvolvem. A biomassa produzida em LPBS dificilmente tem uma concentração maior que $200 \mathrm{mg} \cdot \mathrm{L}^{-1} \mathrm{e}$ a proporção N/SVS em algas é menor que $5 \%$, de modo que a incorporação de $\mathrm{N}$ é menos que $10 \mathrm{mg} \cdot \mathrm{L}^{-1}$. Nitrificação não se desenvolve na LPBS: mesmo com a concentração de OD maior que a saturação, não se detecta traços de nitrito nem de nitrato no efluente das LPBS. Pela mesma razão de abundância de $\mathrm{OD}$, o processo de desnitrificação não pode se desenvolver.

Pelo fato de a taxa de dessorção se tornar lenta quando o $\mathrm{pH}$ chega a um valor de 7,5 (VAN HAANDEL; VAN DER LUBBE, 2019), a remoção de $\mathrm{CO}_{2}$ por dessorção pouco contribui para a remoção de nutrientes, que precisa de um $\mathrm{pH}$ na faixa de 8,5 para a remoção de amônia e 9,7 para a remoção de fósforo. Portanto, para que haja a remoção de nutrientes, é necessário ocorrer a remoção biológica de $\mathrm{CO}_{2}$ pelo processo de fotossíntese.

\section{CONCLUSÕES}

Foi possível estabelecer que a transferência dos compostos voláteis: amônia, dióxido de carbono e oxigênio dissolvido em lagoas de tratamento é adequadamente descrita pela lei de Fick, que afirma que a taxa de transferência é proporcional ao déficit de determinado constituinte, o que corresponde à diferença entre a concentração do composto e seu valor de saturação.

Observou-se que a constante de transferência é inversamente proporcional à profundidade da lagoa e depende da temperatura, de acordo com a equação de Arrehnius. Os seguintes valores numéricos foram determinados em lagoas pe- quenas, agitadas suavemente para uniformizar a concentração na fase líquida:

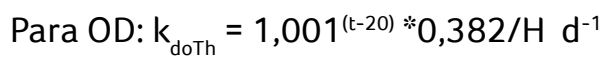

Para $\mathrm{CO}_{2}: \mathrm{k}_{\mathrm{dcTh}}=1,05^{(\mathrm{t}-20) * 0,563 / \mathrm{H} \mathrm{d}^{-1}}$

Para $\mathrm{NH}_{3}: \mathrm{k}_{\mathrm{dcTh}}=0,198^{(\mathrm{t}-20) * 1,095 / \mathrm{H} \mathrm{d}^{-1}}$

Para concentrações muito acima da concentração de saturação, os componentes voláteis tendem a se desprender, formando bolhas na fase líquida. A validade da lei de Fick se limita às seguintes concentrações máximas: $\mathrm{OD}<35 \mathrm{mg} / \mathrm{L} ; \mathrm{CO}_{2}<40$ $\mathrm{mg} / \mathrm{L}$ e $\mathrm{NH}_{3}<60 \mathrm{mg} / \mathrm{L}$. Esses valores são maiores que as concentrações normalmente encontradas em esgoto e, portanto, a lei de Fick descreve a transferência dos compostos adequadamente.

Sob condições normais de operação de LPBS (profundidade $<1 \mathrm{~m}$; DBO $<50 \mathrm{mg} \cdot \mathrm{L}^{-1}$ ), a absorção de oxigênio é importante somente nos primeiros dias de operação; depois, a biomassa de algas se estabelece e sua taxa de produção de OD é maior que a taxa de absorção.

Em LPBS a dessorção de amônia é o mecanismo predominante de remoção de nitrogênio. Incorporação de nitrogênio na biomassa que se forma tem uma pequena contribuição, enquanto nitrificação e desnitrificação não se desenvolvem.

Dessorção de dióxido de carbono é um processo relativamente rápido que se desenvolve principalmente no primeiro dia da operação de LPBS.

\section{AGRADECIMENTOS}

Os autores agradecem ao Conselho Nacional de Desenvolvimento Científico e Tecnológico (CNPq) pelo incentivo financeiro as pesquisas.

\section{CONTRIBUIÇÃO DOS AUTORES}

Todos os autores contribuíram de forma igualitária. 


\section{REFERÊNCIAS}

BUCHAUER, K. A comparison of two simple titration procedures to determine volatile fatty acids in influents to waste-water and sludge treatment processes. Water Sa-Pretoria, v. 24, p. 49-56, 1998.

CAVALCANTI, P. F. F.; VAN HAANDEL, A. C.; LETTINGA, G. Effect of carbon dioxide and ammonium removal on $\mathrm{pH}$ changes in polishing ponds. Water Sci. Technol. v. 45, n. 10, p. 377-382. 2002. https://doi.org/10.2166/wst.2002.0372

CAVALCANTI, P. F. F. Aplicação de reatores UASB e lagoas de polimento no tratamento de esgoto doméstico. ed. Gráfica Santa Marta, João Pessoa, Paraíba. 2009. 172p.

KLOCK, J. W. Survival of coliform bacteria in wastewater lagoons. J Water Pollut Control Fed, v. 50 n. 11, p. 2071-2083, 1971.

PIVELI, R.P.; KATO, M. T. Qualidade das águas e poluição: aspectos físico-químicos. ABES. 2006.

SANTOS, S. L; VAN HAANDEL, A. Variação do pH e remoção de nitrogênio em lagoas de polimento. Revista DAE: Edição especial, São Paulo, n. 229, março 2021.
VAN HAANDEL A. C.; VAN DER LUBBE J. Anaerobic sewage digestion: Theory and applications. International Water Association Londres RU. 2019.

VAN HAANDEL, A.; MARAIS, G. 0 Comportamento do Sistema de Lodo Ativado: Teoria e Aplicações para Projetos e Operação. Campina Grande, Epgraf, 1999. 488p.

VAN HAANDEL, A. C.; LETTINGA, G. Tratamento anaeróbio de esgotos: um manual para regiões de clima quente. Campina Grande, Epgraf, 1994, 220 p.

VON SPERLING, M. Performance evaluation e mathematical modelling of coliform die-off in tropical e subtropical waste stabilisation ponds, Water Research, v. 33, n. 6, p. 1435-1448. 1999. https://doi.org/10.1016/S0043-1354(98)00331-5

YVON-DUROCHER G.; ALLEN A. P.; BASTVIKEN D.; CONRAD, R.; GUDASZ C.; PIERRE, A.; THANH-DUC, N.; DEL GIORGIO, P. A. Methane fluxes show consistent temperature dependence across microbial to ecosystem scales. Nature, v. 507, p. 488-491. 2014. https://doi.org/10.1038/nature13164 